\title{
A prize for discoveries past, present and future
}

\author{
Deep learning has revolutionized the technology industry, but beyond eye-catching applications such as virtual \\ assistants, recommender systems and self-driving cars, deep learning is also transforming many scientific fields.
}

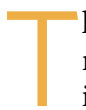

his year's A. M. Turing Award, recognized as the highest distinction in computer science, has been won by three pioneers of deep learning, Geoffrey Hinton, Yoshua Bengio and Yann LeCun. The award is announced annually by the Association for Computing Machinery and comes with a Google-sponsored prize of US\$1 million. The topic chosen for the Turing award this year is likely to be more widely known than most years - with the notable exception of 2016 when the inventor of the World Wide Web, Sir Tim BernersLee, was honoured with the prize. But many will also have heard of deep learning, which can be found in everyday applications using speech recognition, computer vision and language translation. Moreover, deep learning is viewed as an essential component in the long-term strategy towards building artificial intelligence. Alan Turing, after whom the award is named, laid down essential foundations for the field of artificial intelligence and it is certainly fitting that the award has now been presented to those who have made AI mainstream.

In the near term, deep learning is already transforming many scientific areas, especially where high volumes of rich data are available. Certain longstanding questions and challenges may be tackled with well-thought-out implementations of deep learning algorithms, for instance in the search for clean energy sources. One area that looks set to benefit from applying deep learning is nuclear fusion. Tokamak reactors, which confine a high-temperature plasma with large magnetic fields to produce nuclear fusion, could provide a sustainable source of energy. However, practical implementation is obstructed by hard-to-predict plasma instabilities that lead to substantial damage. Scientists recently developed a combination of recurrent and convolutional deep neural networks that were trained on experimental data from nuclear reactors in the United States and Europe and could be taught to forecast disruptions, even those in reactors on which the algorithm was not trained (J. KatesHarbeck, A. Svyatkovskiy and W. Tang, Nature 568, 526-531; 2019).

Deep learning is also regularly employed to address fundamental biological challenges

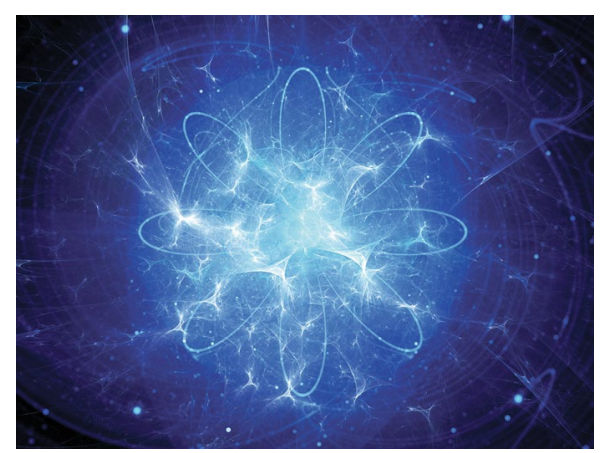

Credit: sakkmesterke/Alamy Stock Photo

such as predicting which part of a protein's amino acid sequence contributes to its biological function, a topic studied in an Article by Dominik Niopek and colleagues in this issue. With 20 different types of amino acids available in nature, there are in theory $20^{n}$ possible proteins of a given length $n$. The potential for guiding exploration in this space to identify proteins with possible biological and medical uses is largely unmet.

Another biological application is in genetic sequencing, where the use of massive throughput techniques calls for highperformance and efficient computational techniques. For instance, in an Analysis, Yi-Ping Phoebe Chen and colleagues compare the performance of several stateof-the-art deep learning approaches in the challenging task of identifying noncoding RNA. While the functionality of most coding RNA is known, it is still an outstanding challenge to understand what role non-coding RNA molecules play in biological processes. An important step is to be able to distinguish between the different molecules in sequencing data, a task well suited to deep learning techniques.

The Turing prize winners are optimistic about the opportunities offered by their discoveries, in scientific applications and elsewhere, but are also well aware of serious ethical concerns around the use of the technique. Even with good intentions, deep learning applications can have unwanted and unfair outcomes considering that the underlying data nearly always has some bias. Deep learning is a powerful tool, especially for those with the computing resources and means to gather large amounts of data, and regulation has not kept up. The need for ethical oversight is widely acknowledged and indeed several reports and guidelines have emerged, most recently 'Ethics guidelines for trustworthy AI' by a highlevel expert group set up by the European Commission. The 'Montréal declaration for a responsible development of artificial intelligence, developed by the Université de Montréal, where Yoshua Bengio is a professor, was presented at the Conference on Neural Information Processing Systems in December last year. And there are plenty more: Algorithm Watch recently held an inventory and counts nearly 60 reports on AI ethics guidelines.

Some commentators warn that it is not realistic to expect industries to self-regulate and even that writing these reports is, in a way, delaying a call for action and regulation. However, the questions involved are complex and it seems appropriate to define principles that are rooted in a commitment to protecting human rights, as is attempted in the European report. Following these principles through, it should become an option to seriously question whether an AI system should be designed at all. It is one of the recommendations of another recent report, from the AI Now Institute, that thorough risk assessments should be applied to AI systems, which should be foregone if the risk of bias and discrimination is substantial. A related point is raised in a Perspective in this issue by Cynthia Rudin, who argues that using deep learning 'black box' models is not warranted in many cases and that attempts to 'open the black box' are futile: instead, interpretable models are needed when peoples' lives are at stake.

The Turing prize is also called the 'Nobel prize' for computing. It seems well within the realm of possibilities that deep learning will lead to scientific breakthroughs that can address some of the world's major challenges. The emphasis should firmly be on deploying deep learning with benefits for all.

Published online: 13 May 2019

https://doi.org/10.1038/s42256-019-0054-Z 